Original contribution

\title{
A new class of cubic SPIONs as a dual-mode T1 and T2 contrast agent for MRI
}

\author{
Akbar Alipour ${ }^{\mathrm{a}}$, Zeliha Soran-Erdem ${ }^{\mathrm{a}, 1}$, Mustafa Utkur ${ }^{\mathrm{a}}$, Vijay Kumar Sharma ${ }^{\mathrm{a}, \mathrm{c}}$, Oktay Algin ${ }^{\mathrm{a}, \mathrm{b}}$, \\ Emine Ulku Saritas ${ }^{\mathrm{a}, \mathrm{d}}$, Hilmi Volkan Demir ${ }^{\mathrm{a}, \mathrm{c}, *}$ \\ ${ }^{a}$ Department of Electrical and Electronics Engineering-National Magnetic Resonance Research Center (UMRAM), National Nanotechnology Research Center (UNAM), \\ Department of Physics, Bilkent University, Bilkent, Ankara 06800, Turkey \\ b Department of Radiology, Ankara Atatürk Training and Research Hospital, Ankara 06800, Turkey \\ ${ }^{\mathrm{c}}$ LUMINOUS! Center of Excellence for Semiconductor Lighting and Displays, School of Electrical and Electronic Engineering, School of Mathematical and Physical Sciences, \\ Nanyang Technological University, Singapore 639798, Singapore \\ d Neuroscience Program, Sabuncu Brain Research Center, Bilkent University, Ankara 06800, Turkey
}

\section{A R T I C L E I N F O}

\section{Keywords:}

Dual-mode MRI contrast agents

Superparamagnetic iron oxide nanoparticles MRI relaxivity

Contrast enhancement

\begin{abstract}
A B S T R A C T
Superparamagnetic iron oxide nanoparticles (SPIONs) are widely used as a robust negative contrast agent on conventional MRI. In this study, we (a) synthesized a new class of cubic SPIONs as a dual-mode contrast agent in MRI and (b) showed the in-vivo feasibility of these nanaoparticles as a simultaneous positive and negative contrast agent. Relaxation properties and contrast enhancement analysis of the synthesized SPIONs with two different shapes (cubic $v s$. spherical) and three different sizes $7 \mathrm{~nm}, 11 \mathrm{~nm}$, and $14 \mathrm{~nm}$ were investigated to evaluate contrast enhancement in-vitro. In-vivo MRI experiments were performed on a 3T MR scanner, where a healthy anesthetized rat was imaged before, and from 20 to $80 \mathrm{~min}$ after intravenous injection of $1 \mathrm{mg} / \mathrm{kg}$ of contrast agent. Representative transmission electron microscopy (TEM) images of the synthesized nanoparticles reveal that the particles are well dispersed in a solvent and do not aggregate. The in-vitro relaxivity and contrast enhancement analysis show that, among all six SPIONs tested, 11-nm cubic SPIONs possess optimal molar relaxivities and contrast enhancement values, which can shorten the spin-lattice and spin-spin relaxation times, simultaneously. No noticeable toxicity is observed during in-vitro cytotoxicity analysis. In-vivo $\mathrm{T}_{1}$-and $\mathrm{T}_{2}$ weighted acquisitions at 60 -min post-injection of $11-\mathrm{nm}$ cubic SPIONs result in $64 \%$ and $48 \%$ contrast enhancement on the $\mathrm{T}_{1}$-and $\mathrm{T}_{2}$-weighted images, respectively. By controlling the shape and size of SPIONs, we have introduced a new class of cubic SPIONs as a synergistic (dual-mode) MRI contrast agent. 11-nm cubic SPIONs with smaller size and high positive and negative contrast enhancements were selected as a promising candidate for dual-mode contrast agent. Our proof-of-concept MRI experiments on rat demonstrate the in-vivo dual-mode contrast enhancement feasibility of these nanoparticles.
\end{abstract}

\section{Introduction}

Superior soft tissue contrast is a major advantage of magnetic resonance imaging (MRI) over alternative medical imaging modalities such as computed tomography (CT) and X-ray $[1,2]$. The difference of spin-lattice $\left(\mathrm{T}_{1}\right)$ and spin-spin $\left(\mathrm{T}_{2}\right)$ relaxation times among tissues, is the origin of the unique soft tissue contrast in MRI. However, in some applications, difference in relaxation parameters may be insufficient to provide reliable discrimination of tissue structure. In these instances, the use of contrast agents that alter relaxivity can significantly enhance the contrast between target tissues and the background. These contrast agents are usually in the form of $\mathrm{T}_{1}$ or $\mathrm{T}_{2}$ contrast agents [3-6]. $\mathrm{T}_{1}$ agents are commonly paramagnetic materials that increase longitudinal relaxivity and thereby yield higher signal levels $[7,8]$. While superparamagnetic iron oxide nanoparticles (SPIONs) with ultrasmall size (e.g., around $36 \mathrm{~nm}$ diameter) can also be used as $\mathrm{T}_{1}$ contrast agents, currently the Gadolinium species with seven unpaired electrons are the dominant positive contrast agent used in clinical applications $[9,10]$. On the other hand, $\mathrm{T}_{2}$ agents are typically SPIONs that induce magnetic field perturbations and increase transverse relaxivity, which in turn reduces signal levels [11-13]. However, the difficulty in distinguishing a region of weak signal due to SPIONs from regions that suffer from

\footnotetext{
* Corresponding author at: Department of Electrical and Electronics Engineering, Bilkent University, Bilkent, 06800 Ankara, Turkey.

E-mail address: Volkan@bilket.edu.tr (H.V. Demir).

${ }^{1}$ This author has equal contribution.
} 
other sources of signal loss (e.g., susceptibility differences among tissues, $B_{0}$ field inhomogeneity) is a drawback of $T_{2}$ contrast agents [14-16]. One solution to this problem is to utilize SPIONs as dualmodal contrast agents. In MRI, $\mathrm{T}_{1}$-weighted images typically provide better spatial resolution, while $\mathrm{T}_{2}$-weighted images can provide enhanced detection of lesions [17]. Hence, a synergistic combination of $\mathrm{T}_{1}$ - and $\mathrm{T}_{2}$-contrast-enhanced imaging can potentially provide more comprehensive imaging information and lead to higher diagnostic accuracy [18].

To date, numerous studies have considered developing methods for synthesizing simultaneous $\mathrm{T}_{1}$ and $\mathrm{T}_{2}$ contrast agents. For this purpose, hybrid nanoparticles have recently been developed, where the Gadolinium species integrated with iron oxide nanoparticles in a coreshell format systematically demonstrated the feasibility of such hybrid probes [19]. In another study, Mn-Fe heterodoped ZnSe tetrapod nanocrystals were proposed to simultaneously enhance contrast in both $\mathrm{T}_{1}$ - and $\mathrm{T}_{2}$-weighted MRI [20]. However, the use of hybrid materials as in these two examples may cause magnetic interactions of nanoparticles, which in turn result in the perturbation of the relaxation process [21]. An alternative method for achieving dual-mode contrast agent is to utilize a single material for enabling multiple contrasts. For example, Jung et al. generated both negative and positive image contrasts by manipulating the pulse sequences together with the use of sizecontrolled SPIONs [22]. In another study, $\mathrm{T}_{1}$-weighted positive contrast obtained from SPIONs using ultrashort-echo-time was reported by Kwon et al. [23]. In the clinic, Ferumoxytol (an ultrasmall SPION) can be utilized to obtain regional $\mathrm{T}_{1}$ and $\mathrm{T}_{2}{ }^{*}$ signal enhancement or loss with conventional pulse sequences [24]. However, SPION's performance as MRI contrast agents strongly depends on their size and shape. Hence, as dual-mode contrast agents are gaining interest, there is a strong need to investigate both features to reach a high performance synergistic contrast agent.

In this study, by taking advantage of the strong shape and size dependence of SPION's performance, we synthesized a new class of cubic SPIONs as a synergistic $\mathrm{T}_{1}$ - and $\mathrm{T}_{2}$-contrast-enhancement agent for MRI. We compared the performance of these cubic SPIONs with their spherical counterparts via measurements of molar relaxivities $\left(r_{1}\right.$ and $\left.r_{2}\right)$ on a 3T MRI scanner. Here, we used silica for surface modification, since previous reports showed negligible toxicity level for nano-sized silica shells. As a feasibility study, both positive and negative contrast effects of 11-nm cubic SPIONs were demonstrated in-vivo on $\mathrm{T}_{1^{-}}$and $\mathrm{T}_{2^{-}}$ weighted spin-echo MRI images obtained before and after intravenous injection of $1 \mathrm{mg} / \mathrm{kg}$ contrast agent trough the tail vein of a SpragueDawley rat.

\section{Materials and methods}

\subsection{Theory}

The magnetic properties of SPIONs are affected by their crystallinity and size, which in turn significantly affects the MR relaxivity properties of the SPIONs. Accordingly, as the particle size increases the corresponding saturation magnetization increases, enhancing the $\mathrm{T}_{2}$ (transverse) relaxation of the surrounding tissue. On the other hand, as the particle size decreases, both the surface-to-volume ratio and the percentage of unpaired electrons on the particle surface increases. The interaction of unpaired electrons with the protons of water molecules reduces the $T_{1}$ (longitudinal) relaxation time of the protons and results in MRI signal enhancement, and this effect is more pronounced for small-sized SPIONs [25]. In addition, the reduced magnetic moments of these smaller particles suppress their $\mathrm{T}_{2}$ effect. Moreover, particles with sharp surfaces (for example cubic particles) possess higher surface-tovolume ratio in comparison with spherical particles, causing them to exhibit improved $\mathrm{T}_{1}$ and $\mathrm{T}_{2}$ effects. Therefore, the shape anisotropy and large effective region of magnetic cores in cubic samples can increase their $\mathrm{T}_{2}$-contrast-enhancement capabilities, and large surface-to- volume ratio and consequently a large number of unpaired electrons on their cubic surface can increase their $\mathrm{T}_{1}$-contrast-enhancement capabilities [26,27].

In the well-known form, $\mathrm{T}_{1}$ and $\mathrm{T}_{2}$ can be represented in terms of their corresponding $R_{1}$ and $R_{2}$ relaxation rates, respectively [28]. The relaxation rates are functions of unenhanced tissue relaxation rates $\left(R_{10}\right.$ and $R_{20}$ ) in $s^{-1}$, longitudinal and transverse Molar relaxivities ( $r_{1}$ and $\mathrm{r}_{2}$ ) in $\mathrm{mM}^{-1} \mathrm{~s}^{-1}$, and iron concentration (C) in $\mathrm{mM}$. Using the standard linear relaxivity approximation, the relaxation rate can be written as:

$\mathrm{R}_{1}=\mathrm{R}_{1_{0}}+\mathrm{r}_{1}$. C

$\mathrm{R}_{2}=\mathrm{R}_{20}+\mathrm{r}_{2} \cdot \mathrm{C}$

where

$\mathrm{R}_{\mathrm{x}}=\frac{1}{\mathrm{~T}_{\mathrm{x}}}$ and $\mathrm{R}_{\mathrm{x} 0}=\frac{1}{\mathrm{~T}_{\mathrm{x} 0}}(\mathrm{x}$ is either 1 or 2$)$

In the current study, the MR contrast optimization is conducted with multi-echo spin-echo sequences and inversion recovery sequences for transverse and longitudinal relaxation estimation, respectively. Under these approximations, the $\mathrm{T}_{1}$-weighted and $\mathrm{T}_{2}$-weighted signals can be estimated by the following equations:

For longitudinal relaxation:

$$
\begin{aligned}
\mathrm{I}_{\mathrm{z}} & =\mathrm{M}_{\mathrm{z}}^{0}\left[1-2 \exp \left\{\frac{-\mathrm{TI}}{\mathrm{T} 1}\right\}+\exp \left\{\frac{-\mathrm{TR}}{\mathrm{T} 1}\right\}\right. \\
& =\mathrm{M}_{\mathrm{z}}^{0}\left[1-2 \exp \left\{-\mathrm{TI}\left(\mathrm{R}_{1_{0}}+\mathrm{r}_{1} \cdot \mathrm{C}\right)\right\}+\exp \left\{-\mathrm{TR}\left(\mathrm{R}_{1_{0}}+\mathrm{r}_{1} \cdot \mathrm{C}\right)\right\}\right.
\end{aligned}
$$

and for transverse relaxation:

$\mathrm{I}_{\mathrm{xy}}=\mathrm{M}_{\mathrm{z}}^{0} \exp \left\{\frac{-\mathrm{TE}}{\mathrm{T}_{2}}\right\}=\mathrm{M}_{\mathrm{z}}^{0} \exp \left\{-\mathrm{TE}\left(\mathrm{R}_{2_{0}}+\mathrm{r}_{2} \cdot \mathrm{C}\right)\right\}$

Here, TE is the echo time, TI is the inversion time, TR is the repetition time, and $\mathrm{M}_{\mathrm{z}}{ }^{0}$ is the thermal equilibrium value of bulk magnetization vector. TE and TI are image acquisition parameters controlled by the operator. $\mathrm{T}_{1}$ and $\mathrm{T}_{2}$ depend on the surrounding medium and the magnetic field strength.

As the contrast agents shorten the spin-lattice and spin-spin relaxation times, the ratio of their molar relaxivities $\left(\alpha=r_{2} / r_{1}\right)$ is generally used to describe the dominant contrast agent features and their contrast enhancement efficiency [29]. Accordingly, $\alpha$ is a parameter comparing the effectiveness of $\mathrm{T}_{1}$ and $\mathrm{T}_{2}$ contrast mechanisms, and this parameter strongly depends on particle properties, contrast regime, and $\mathrm{B}_{0}$ field strength [30].

\subsection{Materials}

For the synthesis of SPIONs, the following materials were obtained from Sigma-Aldrich Chemical Co. (St. Louis, MO): oleic acid (tech $90 \%$ ), iron(III) chloride hexahydrate (99.99\%), Igepal-CO520 (polyoxyethylene (5) nonyl phenyl ether), ammonia (28 wt\%), tetraethyl orthosilicate (TEOS 99\%) and 1-octadecene (tech 90\%). Hexane, sodium hydroxide, cyclohexane, ethanol and other reagents were obtained from Alfa Aesar Chemical Co. (Germany). All other chemicals were of analytic grade and used directly without further purification.

\subsection{Synthesis of SPIONs}

In this work, six different types of SPION samples were synthesized: 7-nm spherical, 7-nm cubic, 11-nm spherical, 11-nm cubic, 14-nm spherical, and 14-nm cubic. For the cubic nanoparticles, nanoparticle size was measured diagonally. For all samples, iron oxide nanoparticles were synthesized using a modified procedure reported by Byung et al. [15]. Briefly, $20 \mathrm{~g}$ (25 mL) of 1-octadecene (ODE) solution, oleic acid and freshly synthesized iron-oleate were stirred in a three-neck bottle 

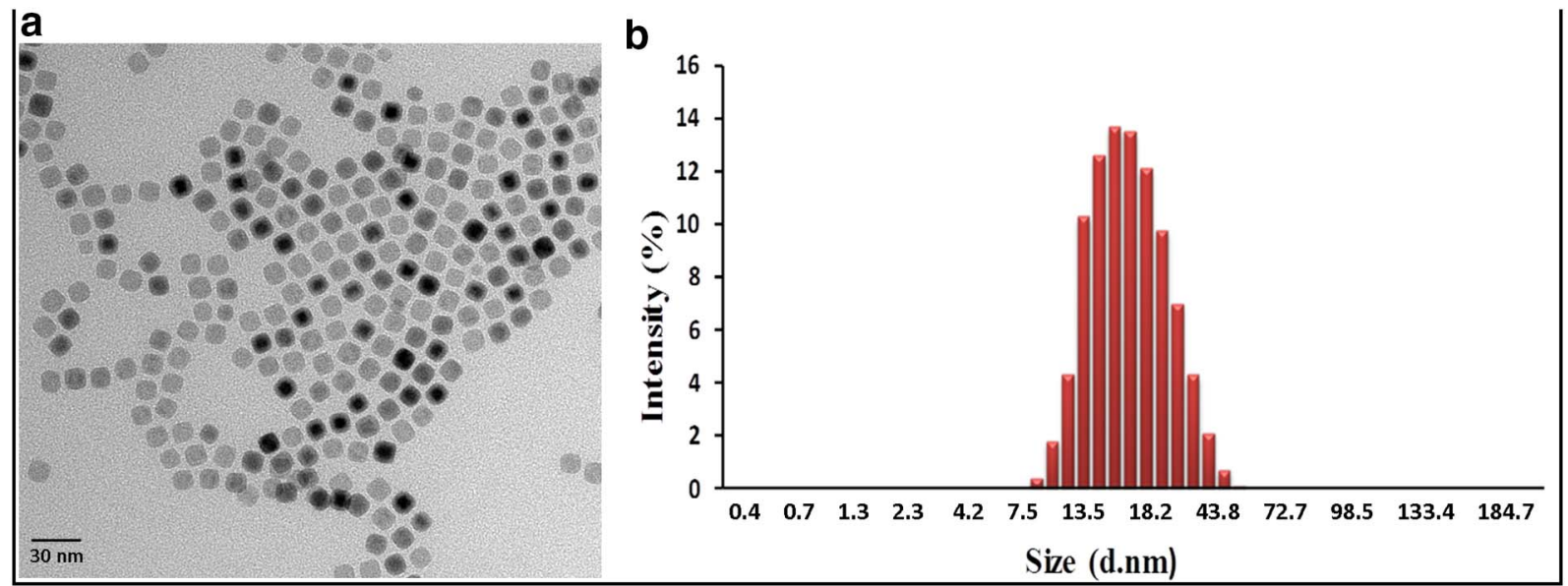

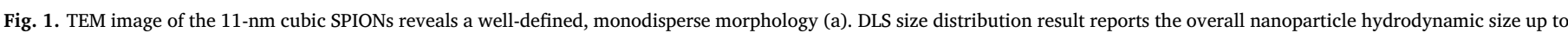
$\sim 18 \mathrm{~nm}$ (b).

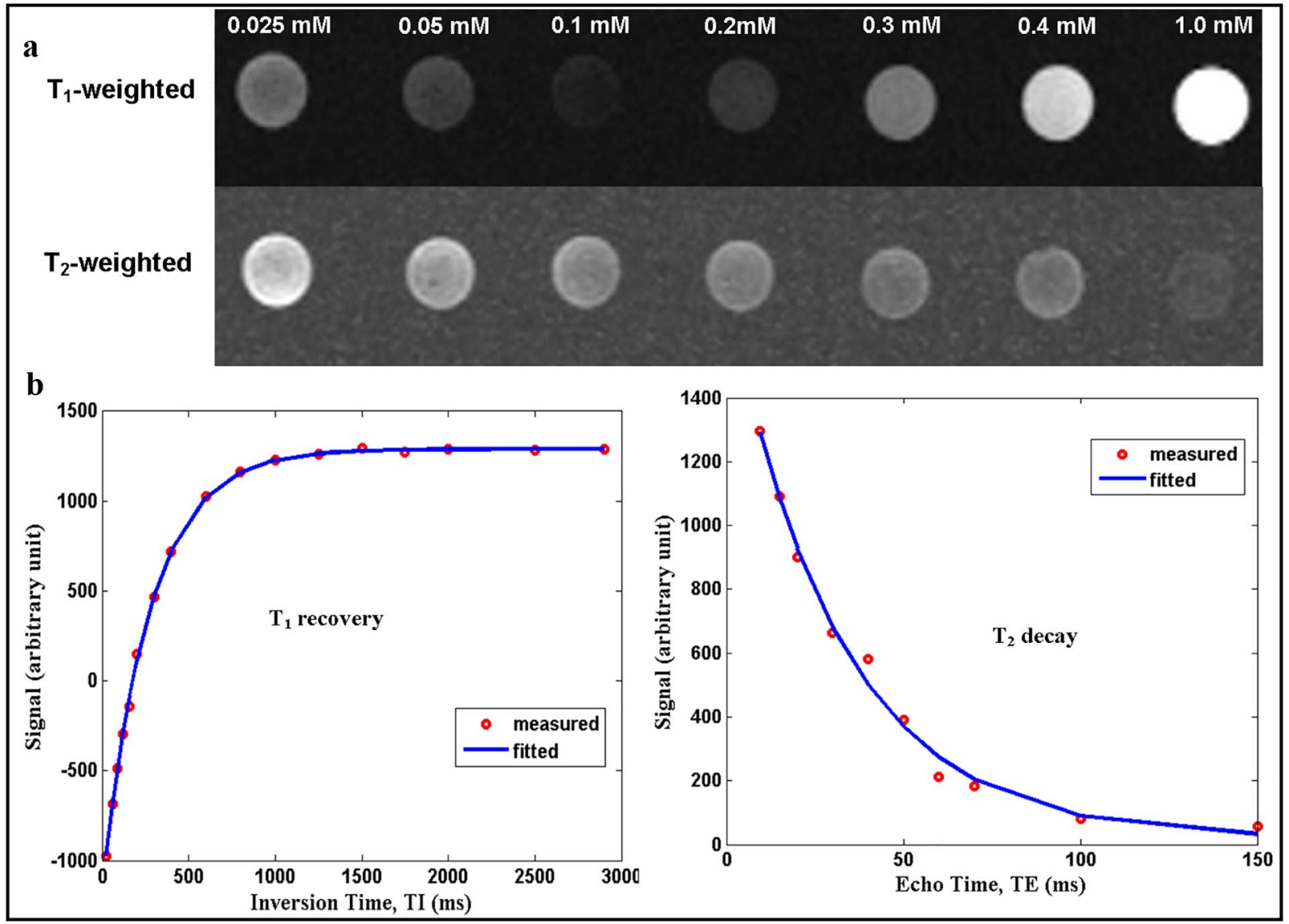

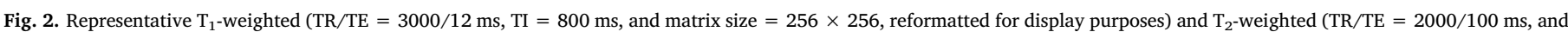

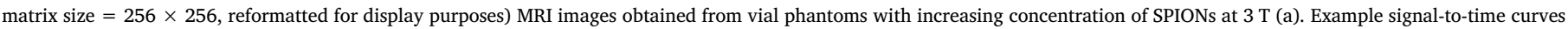

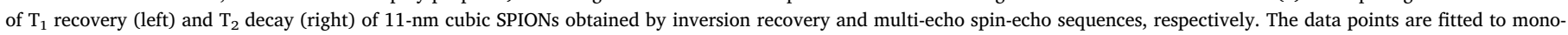
exponential functions given in Eqs. (3) and (4) to estimate $\mathrm{T}_{1}=500 \mathrm{~ms}$ and $\mathrm{T}_{2}=35 \mathrm{~ms}$ values for 11-nm cubic SPIONs at $0.4 \mathrm{mM}$ concentration (b).

flask and degassed at $70{ }^{\circ} \mathrm{C}$ for 2 -hour under vacuum. Then, the temperature of the reaction mixture was increased to $320^{\circ} \mathrm{C}$ with constant heating rates of $3.3{ }^{\circ} \mathrm{C} \mathrm{min}-1,4.2^{\circ} \mathrm{C} \mathrm{min}^{-1}$ or $5.5^{\circ} \mathrm{C} \mathrm{min}{ }^{-1}$ under argon flow. The growth of the SPIONs was done at $320^{\circ} \mathrm{C}$ for $30 \mathrm{~min}$. Following the growth, the solution was cooled down to room temperature and SPIONs were cleaned with 2-propanol and acetone for three times. Then, SPIONs were dispersed in $16 \mathrm{~mL}$ of hexane and filtered using $0.2 \mu \mathrm{m}$ Millipore filter. In this work, we achieved the differences in the size and shape of SPIONs by varying the oleic acid amount, heating rate, and iron-oleate amount of the mixture.

\subsection{Surface modification}

Principally, nanoparticles must possess low toxicity and good colloidal stability in a biological environment to be suitable for in-vivo applications, which mandates a surface modification of these nanoparticles. Formation of biocompatible shells surrounding the nanoparticles is a typical method for surface modification. Briefly, $10 \mathrm{~mL}$ of 

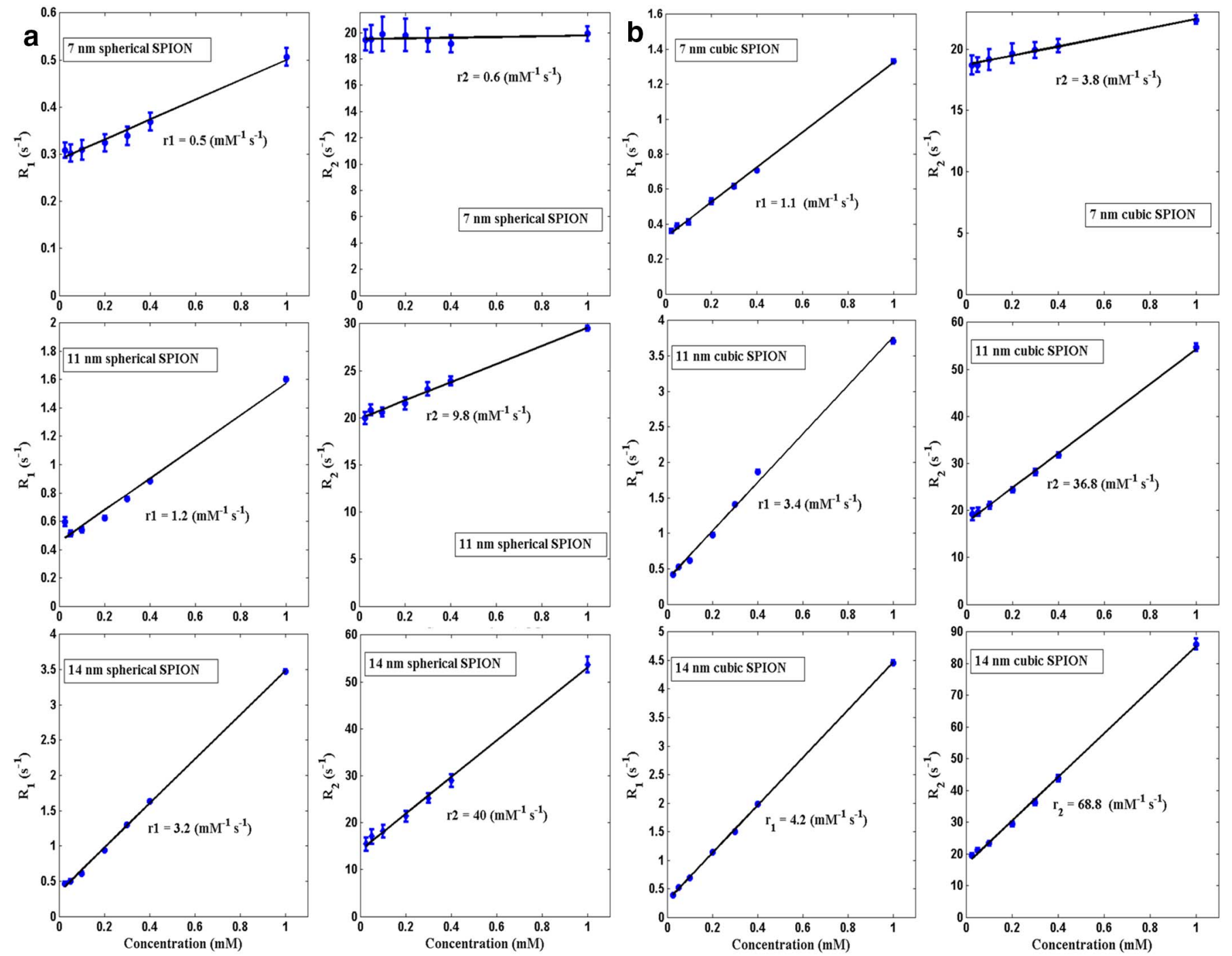

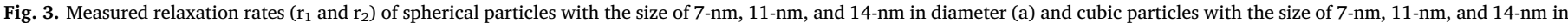

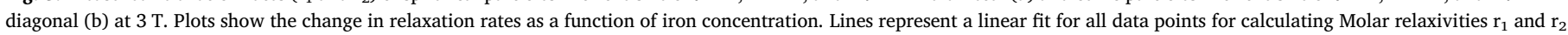

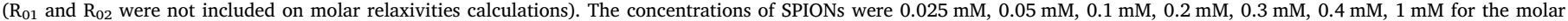
relaxivities measurement.

Table 1

Molar relaxivities measured at $3 \mathrm{~T}$.

\begin{tabular}{lllllll}
\hline \multicolumn{2}{c}{ Group A spherical SPION } & & \multicolumn{5}{c}{ Group B cubic SPION } \\
\hline Particle & $7 \mathrm{~nm}$ & $11 \mathrm{~nm}$ & $14 \mathrm{~nm}$ & $7 \mathrm{~nm}$ & $11 \mathrm{~nm}$ & $14 \mathrm{~nm}$ \\
$\mathrm{r}_{1}\left(\mathrm{mM}^{-1} \mathrm{~s}^{-1}\right)$ & 0.5 & 1.2 & 3.2 & 1.1 & 3.4 & 4.2 \\
$\mathrm{r}_{2}\left(\mathrm{mM}^{-1} \mathrm{~s}^{-1}\right)$ & 0.6 & 9.8 & 40 & 3.8 & 36.8 & 68.8 \\
$\alpha=\mathrm{r}_{2} / \mathrm{r}_{1}$ & 1.2 & 8.2 & 12.5 & 3.4 & 10.8 & 16.4 \\
\hline
\end{tabular}

cyclohexane and $1.3 \mathrm{~mL}$ of Igepal-CO520 (polyoxyethylene (5) nonyl phenyl ether) were stirred for $15 \mathrm{~min}$ to obtain a microemulsion dispersion. Followed by the addition of $1.5 \mathrm{mg}$ iron oxide nanoparticles (in $1.6 \mathrm{~mL}$ of cyclohexane), the mixture was continuously stirred for further $15 \mathrm{~min}$. Subsequently, $40 \mu \mathrm{L}$ of TEOS was added, and the solution was stirred for $30 \mathrm{~min}$ in the dark. Finally, $150 \mu \mathrm{L}$ of ammonium hydroxide $(1.5 \%)$ solution was added, and the mixture was stirred for $72 \mathrm{~h}$. For the cleaning of resulting silica-coated iron oxide nanoparticles, the solution was centrifuged at $10000 \mathrm{rpm}$ for $20 \mathrm{~min}$. After discarding the supernatant, $10 \mathrm{~mL}$ of ethanol was added, and the nanoparticles were dispersed using an ultrasonic bath. This dispersion was centrifuged at $10000 \mathrm{rpm}$ for $40 \mathrm{~min}$, and the same procedure was repeated one more time. At the end, the supernatant was removed, and
$6 \mathrm{~mL}$ of autoclaved $\mathrm{ddH}_{2} \mathrm{O}$ and $24 \mu \mathrm{L}$ of $2 \mathrm{M}$ sodium hydroxide $(\mathrm{NaOH})$ solution were added.

\subsection{Characterization}

Morphological structures of the iron oxide nanoparticles were investigated using an FEI Tecnai G2 F30 transmission electron microscopy (TEM). Before imaging, $2 \mu \mathrm{L}$ of iron oxide nanoparticle solution in hexane was dropped on a 200 mesh copper grid, and the sample was dried. Dynamic light scattering (DLS) measurement was conducted to study nanoparticles size distributions.

\subsection{In-vitro relaxivity measurement}

For in-vitro relaxivity analysis, solutions of all six types of SPIONs in varying concentrations were prepared in hexane to measure at 3T MRI scanner (Siemens Magnetron Trio, $f_{o}=123 \mathrm{MHz}$ ) using a 32-channel receive-only head coil (Siemens). The medium containing SPIONs was systematically diluted with hexane over the range of concentrations $(0.025-1 \mathrm{mM})$ in glass vials $(10 \mathrm{~mm}$ in diameter and $20 \mathrm{~mm}$ in height). The vials were placed vertically within the head coil. Experiments were performed at room temperature. $\mathrm{T}_{1}$ values were measured using an 


\begin{tabular}{|c|c|c|c|c|c|c|}
\hline Sample & $\mathrm{T}_{1}-\mathrm{W}$ MRI image & $\mathrm{T}_{2}$-W MRI image & $\mathrm{C}_{\text {Enh }}\left(\mathrm{T}_{1}-\mathrm{W}\right) \%$ & $\mathrm{C}_{\text {Enh }}\left(\mathrm{T}_{2}-\mathrm{W}\right) \%$ & $\operatorname{SNR}\left(T_{1}-W\right)$ & $\operatorname{SNR}\left(T_{2}-W\right)$ \\
\hline $\begin{array}{c}\text { Reference Phan- } \\
\text { tom }\end{array}$ & & & 0 & 0 & 8.1 & 92.5 \\
\hline 7-nm Sphere & & & 570 & 70 & 10.9 & 15.6 \\
\hline 11-nm Sphere & & & 650 & 74 & 16.2 & 11.4 \\
\hline 14-nm Sphere & & & 990 & 89 & 28.5 & 6.0 \\
\hline 7-nm Cubic & & & 650 & 53 & 14.9 & 23.6 \\
\hline 11-nm Cubic & & & 940 & 85 & 28.1 & 8.1 \\
\hline 14-nm Cubic & & & 790 & 89 & 23.4 & 5.9 \\
\hline
\end{tabular}

Fig. 4. In-vitro contrast enhancement analysis of the synthesized SPIONs. SNR and $\mathrm{C}_{\mathrm{Enh}}$ calculation results show that 11-nm cubic and 14-nm spherical particles have better dual-mode contrast enhancement behavior. Six different samples with $0.4 \mathrm{mM}$ concentration were utilized for $\mathrm{T}_{1}$ and $\mathrm{T}_{2}$-weighted $\mathrm{MRI}$ imaging. $\mathrm{T}_{1}$-weighted images were obtained using inversion recovery sequences: $\mathrm{TR}=3000 \mathrm{~ms}, \quad \mathrm{TE}=12 \mathrm{~ms} ， \quad \mathrm{TI}=700 \mathrm{~ms} . \quad \mathrm{T}_{2}$-weighted images were obtained using multi-echo spin-echo sequences: $\mathrm{TR}=2000 \mathrm{~ms}$, TE $=100 \mathrm{~ms}$. Here, the gray scale display windowing was adjusted to better display the differences between signal levels.
Table 2

Contrast enhancement analysis results of the in vivo experiment.

SNR: signal-to-noise ratio, CNR: contrast-to-noise ratio, $\mathrm{C}_{\mathrm{Enh}}$ : contrast enhancement.

\begin{tabular}{|c|c|c|c|c|c|c|}
\hline \multirow[b]{2}{*}{ Imaging timeline } & \multicolumn{3}{|c|}{$\mathrm{T}_{1}$-weighted } & \multicolumn{3}{|c|}{$\mathrm{T}_{2}$-weighted } \\
\hline & SNR & CNR & $\mathrm{C}_{\mathrm{Enh}}(\%)$ & SNR & CNR & $\mathrm{C}_{\mathrm{Enh}}(\%)$ \\
\hline Pre-contrast & 34.9 & 6.3 & - & 22.2 & 12.6 & - \\
\hline 5 min post-contrast & 45.7 & 17.2 & 31 & 18.8 & 9.2 & 15 \\
\hline 35 min post-contrast & 50.6 & 20.1 & 45 & 14.4 & 4.9 & 35 \\
\hline 70 min post-contrast & 57.4 & 22.3 & 64 & 11.4 & 1.8 & 48 \\
\hline 90 min post-contrast & 56.8 & 21.9 & 61 & 11.8 & 2.0 & 47 \\
\hline 18 -hour post-contrast & 37.3 & 9.2 & 6 & 20.0 & 10.6 & 1 \\
\hline
\end{tabular}

inversion recovery sequence: $\mathrm{TI}=[24,60,90,120,200,400,800$, $1250,1750,2000,2500,2900] \mathrm{ms}, \mathrm{TR}=3000 \mathrm{~ms}, \mathrm{TE}=12 \mathrm{~ms} . \mathrm{T}_{2}$ values were measured using a multi-echo spin-echo sequences: with $\mathrm{TE}=[9,15,20,30,40,50,60,70,100,150] \mathrm{ms}, \mathrm{TR}=2000 \mathrm{~ms}$. Other parameters were kept the same for both sequences: slice thickness $=3 \mathrm{~mm}$, field-of-view $\quad(\mathrm{FOV})=74 \times 149 \mathrm{~mm}, \quad$ matrix si$\mathrm{ze}=256 \times 256$ pixels.

MR data were quantitatively analyzed off-line with the use of an inhouse software developed in MATLAB (The MathWorks, Natick, MA). A circular region of interest (ROI) was outlined within each vial. For a given pixel within a ROI, the signal intensities at all TI values were gathered, and the $\mathrm{T}_{1}$ relaxation time was determined via a LevenbergMarquardt nonlinear fitting of these signal intensities to Eq. (3). This was repeated for all pixels within the ROI, and the mean and standard deviation of $\mathrm{T}_{1}$ was computed across all pixels in the ROI. A similar procedure was performed for $\mathrm{T}_{2}$ measurement, this time gathering the signal intensity for a given pixel at all TE values, and nonlinear fitting these intensities to Eq. (4). The relaxation rates $R_{1}$, and $R_{2}$ were calculated from the estimated $\mathrm{T}_{1}$ and $\mathrm{T}_{2}$ values. Finally, Molar relaxivities $r_{1}$ and $r_{2}$ were quantified by a linear fitting of the $R_{1}$ or $R_{2}$ values to Eqs. (1) and (2), respectively.

\subsection{Cytotoxicity}

As recommended by ISO 10993-5:2009, we used L929 mouse cell line to investigate the in-vitro cellular toxicity of $11-\mathrm{nm}$ silica-coated cubic SPIONs. The cell line was grown in Dulbecco's modified Eagle's media (DMEM) solution containing 10\% fetal bovine serum (FBS). For analysis of cellular visibility, the cell line was cultured in a 96-well plate $(\mathrm{n}=3$ ) with different concentrations of 11-nm silica-coated cubic SPIONs. An incubation time of $24 \mathrm{~h}$ was utilized [11,12]. After incubation, the $200 \mu \mathrm{L}$ of supernatant was transferred into the 96-well plate to mix with Alamar Blue solution. After several repetitions, $10 \mu \mathrm{L}$ of the mixed solution was picked up to count the number of living cells using automated cell counter.

\subsection{In-vivo MR imaging}

In-vivo MRI experiments were approved by the institutional Animal Ethics Committee and performed by the national standards of animal care. Sprague Dawley rat (250-300 g) was obtained from institutional Animal Laboratory and was maintained in standard laboratory conditions. In-vivo MRI studies were conducted with 3T MRI scanner. The rat 


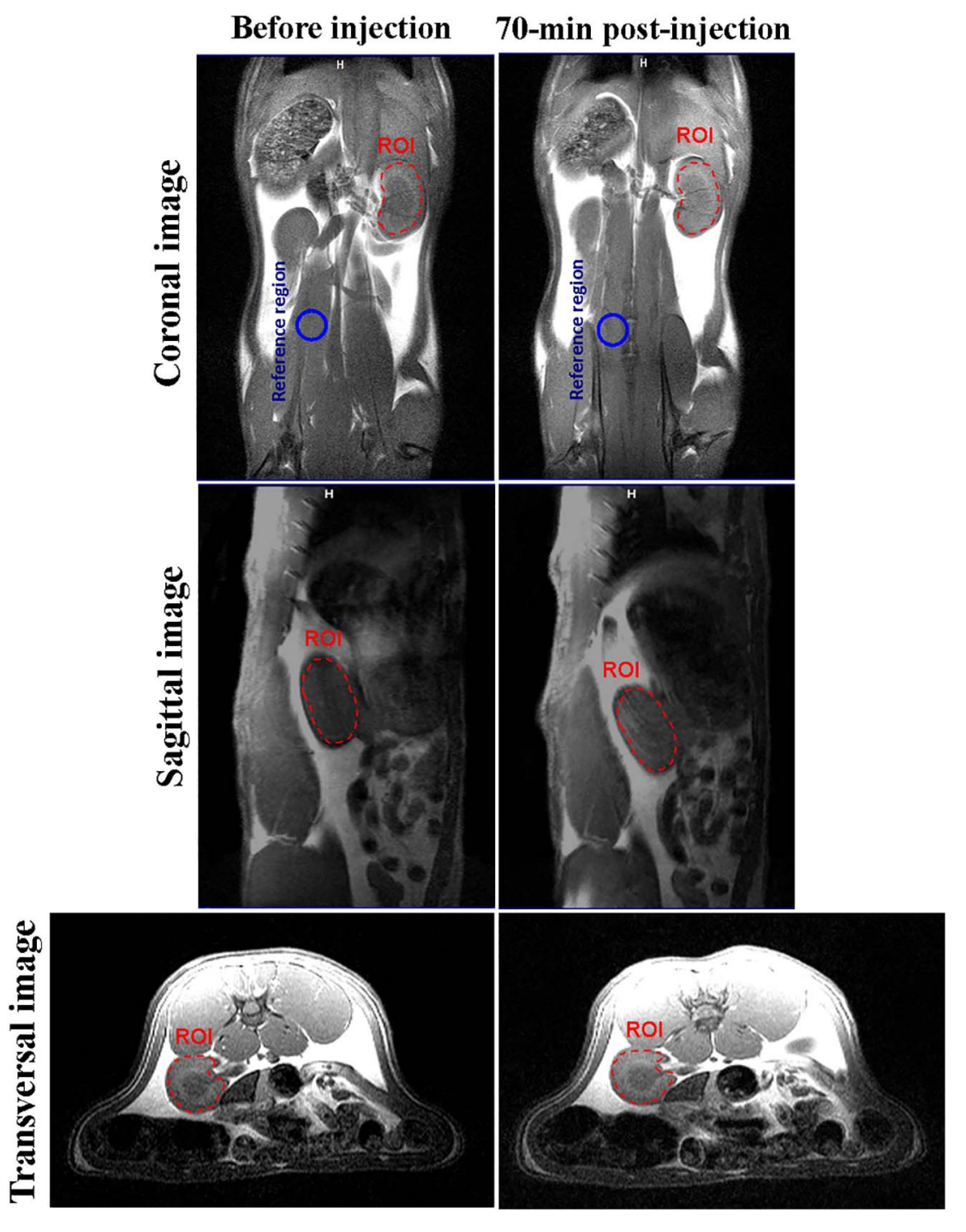

Fig. 5. In-vivo $\mathrm{T}_{1}$-weighted spin-echo $\mathrm{MRI}$ images of a healthy rat, acquired on a 3T MRI scanner. Images were acquired before and 70min post contrast injection of $11-n m$ cubic silica-coated SPIONs. A dose of $1 \mathrm{mg} / \mathrm{kg}$ of contrast agent was injected via the tail vein. Areas of signal enhancement (after injection) indicate approximately $64 \%$ contrast improvement in the kidney (marked with red dashed circles). (For interpretation of the references to colour in this figure legend, the reader is referred to the web version of this article.) was placed headfirst inside an in-house-developed cylindrical 5-cmdiameter birdcage rat MRI coil. This custom receive coil was used for all of our in-vivo experiments.

Due to their small-size advantage and high $\mathrm{T}_{1^{-}}$and $\mathrm{T}_{2}$-contrast-enhancement (see results), 11-nm cubic silica-coated SPIONs were chosen for the in-vivo experiments. MR contrast enhancement simulations were conducted before and after SPION injections at various imaging parameters (TR, TE, and flip angle). The relationships of the signal enhancement with respect to the imaging parameters as function of SPION concentration $(22,23)$ showed that SPION can act as positive and negative contrast agent. With a short TR, short TE, and large flip angle, the signal intensity increased with the increasing SPION concentration, denoting a $\mathrm{T}_{1}$-enhancement effect. Increasing SPION concentration at a long TR values, long TE values, and low flip angles resulted in reduced signal intensity, denoting a $\mathrm{T}_{2}$-enhancement effect.

The aim of the in-vivo experiments was to show the proof-of-concept feasibility of synergistic contrast enhancement feature of the 11-nm cubic SPIONs in-vivo. Our animal experiments did not include an exhaustive analysis of the effects of these nanoparticles on all anatomical organs. Instead, we focused on the kidney as the ROI due to the rapid accumulation of SPIONs in the kidney.

To observe the synergistic contrast effect, healthy anesthetized rat was imaged before, and $5 \mathrm{~min}, 35 \mathrm{~min}, 70 \mathrm{~min}, 90 \mathrm{~min}$, and $18 \mathrm{~h}$ after intravenous injection of $1 \mathrm{mg} / \mathrm{kg}$ 11-nm cubic silica-coated SPIONs. A conventional spin-echo sequence $(\mathrm{TR} / \mathrm{TE}=1000 \mathrm{~ms} / 11 \mathrm{~ms}, 2 \mathrm{~mm}$ slice thickness, and $320 \times 320$ matrix size) was performed to obtain $\mathrm{T}_{1}$ weighted anatomical scans. $\mathrm{T}_{2}$-weighted spin-echo imaging was conducted with the following parameters: TR/TE $=4000 \mathrm{~ms} / 90 \mathrm{~ms}$, $2 \mathrm{~mm}$ slice thickness, and $320 \times 320$ matrix size.

\subsection{Contrast enhancement analysis}

The in-vitro and in-vivo SPION-induced contrast enhancement analysis was performed using calculation of contrast-to-noise ratio (CNR), SNR, and percentage of contrast enhancement $\left(\mathrm{C}_{\mathrm{Enh}}\right)$.

For in-vitro contrast enhancement analysis, $0.4 \mathrm{mM}$ of all six types of SPIONs as well as a reference sample (without contrast material) was prepared on $2 \mathrm{~mL}$ vials. We used inversion recovery (TR $=3000 \mathrm{~ms}$, $\mathrm{TE}=12 \mathrm{~ms}$ and $\mathrm{TI}=600 \mathrm{~ms}$ ) and multi-echo spin-echo ( TR $=2000 \mathrm{~ms}, \mathrm{TE}=100 \mathrm{~ms}$ ) MRI sequences to analyze the $\mathrm{T}_{1}$ - and $\mathrm{T}_{2}$-contrast-enhancement, respectively. In-vivo contrast enhancement analysis was performed on the images resulting from the experiments described in the In-vivo MR imaging section above.

For each image group, SNR analysis was performed to determine the signal variations on the ROI with and without contrast agents. The signal intensities were gathered for all given pixels within a ROI, then mean signal intensity was calculated. The SNR was calculated by dividing mean signal intensity of ROI by standard deviation of noise.

The $\mathrm{C}_{\mathrm{Enh}}$ in the phantom and in-vivo were calculated using the following equation: 


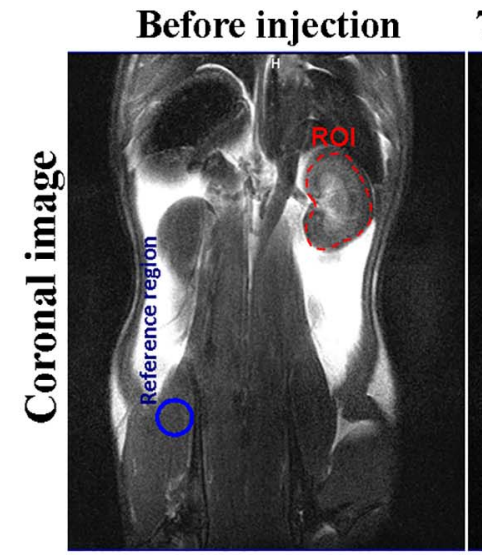

\section{0-min post-injection}
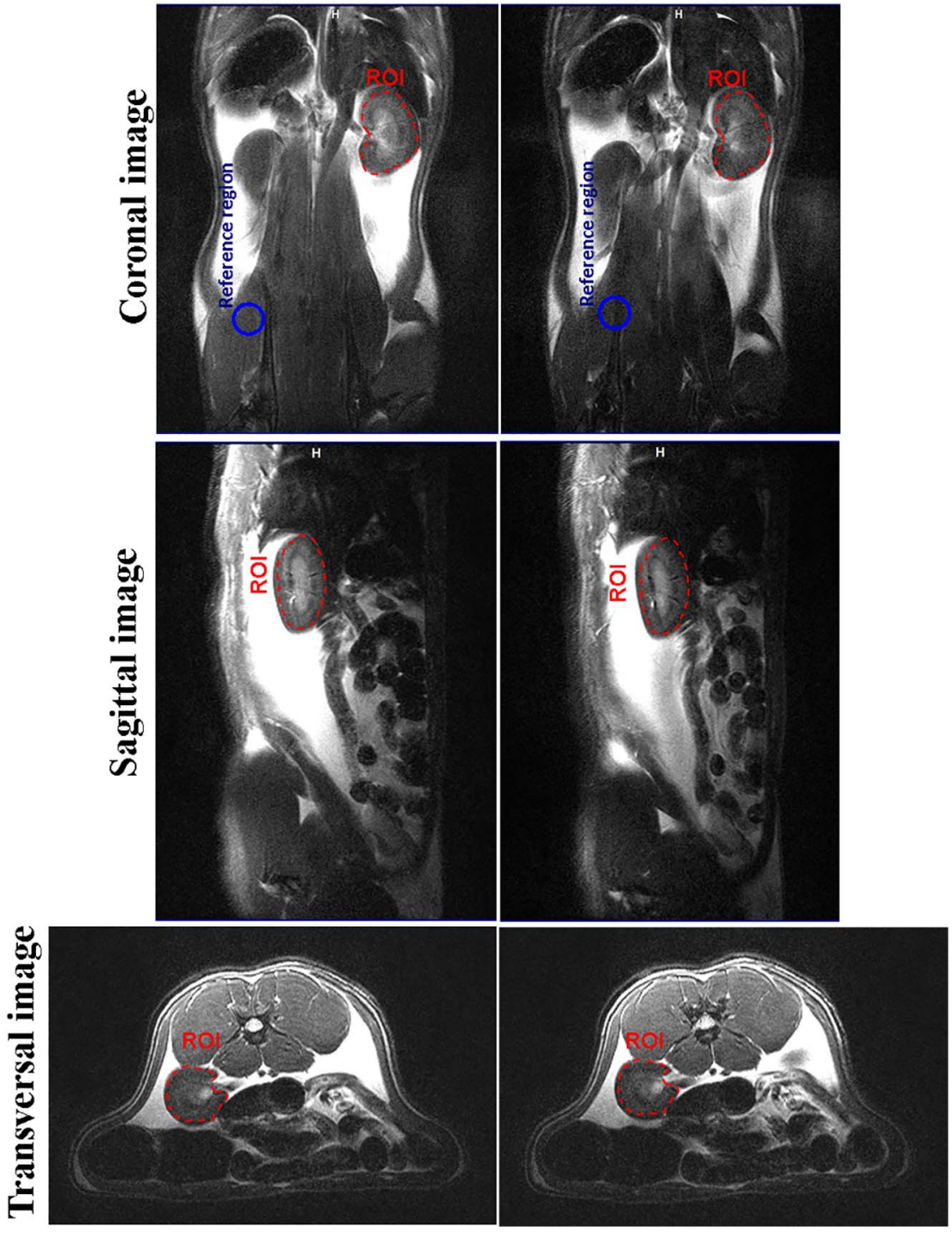

Fig. 6. In-vivo $\mathrm{T}_{2}$-weighted spin-echo $\mathrm{MRI}$ images of a healthy rat, acquired on a 3T MRI scanner. Images were acquired before and 70min post contrast injection of 11-nm cubic silica-coated SPIONs. A dose of $1 \mathrm{mg} / \mathrm{kg}$ of contrast agent was injected via the tail vein. At 70-min post-injection of the contrast agent, the signal in the kidney became noticeably dark and resulted in approximately $48 \%$ contrast enhancement (marked with red dashed circles). (For interpretation of the references to colour in this figure legend, the reader is referred to the web version of this article.)
$\mathrm{C}_{\text {Enh }}=\left|\frac{\mathrm{I}_{\text {post }}-\mathrm{I}_{\text {pre }}}{\mathrm{I}_{\text {pre }}}\right| \times 100$

where $\mathrm{I}_{\text {post }}$ and $\mathrm{I}_{\text {pre }}$ are the mean signal intensities of the ROI with and without SPIONs, respectively.

The CNR was calculated using the following equations:

$\mathrm{CNR}=\left|\frac{\mathrm{I}_{\mathrm{ROI}}-\mathrm{I}_{\mathrm{REF}}}{\sigma_{\mathrm{N}}}\right|$

where $I_{\text {ROI }}$ and $I_{\text {REF }}$ are the mean signal intensities of the ROI and a reference region, respectively. $\sigma_{\mathrm{N}}$ is the standard deviation of noise. For in-vivo contrast enhancement analysis, we focused on the kidneys as the ROI, and psoas minor muscle was selected as the reference region to calculate SNR, CNR, and $\mathrm{C}_{\text {Enh }}$ (Fig. 5, 6).

\section{Results}

\subsection{Synthesis of silica-coated SPION and characterization}

Spherical and cubic SPIONs with the size of $7 \mathrm{~nm}, 11 \mathrm{~nm}$, and $14 \mathrm{~nm}$ were synthesized using the thermal decomposition method. TEM was utilized for the morphological and structural characterizations of iron oxide nanocrystals. Representative TEM images and a comparison of core sizes of the synthesized SPIONs reveal that the particles are monodisperse, well separated in a solvent, and do not aggregate. An example TEM image corresponding to the 11-nm cubic SPIONs is shown in Fig. 1a. DLS measurements of the same SPIONs are given Fig. 1b, showing a hydrodynamic size of up to $\sim 18 \mathrm{~nm}$.

\subsection{In-vitro relaxivity analysis of synthesized SPIONs}

In-vitro $\mathrm{T}_{1}$ and $\mathrm{T}_{2}$ relaxation times of all prepared vial phantoms as a function of the iron concentration were calculated for all samples. The relaxation times were determined by nonlinear fitting of the signal intensities (Fig. 2b). An example $\mathrm{T}_{1}$-weighted image shown in Fig. 2a shows that mean signal intensities of the phantoms first go down and then increase with increasing iron concentration, due to inversion recovery. In the $\mathrm{T}_{2}$-weighted images, by increasing the iron concentration the mean signal intensities decrease. As expected, the relaxation rates $\left(R_{1}\right.$ and $\left.R_{2}\right)$ increase linearly with increasing iron concentration of the nanoparticles. More importantly, the longitudinal and transverse molar relaxivities, $r_{1}$ and $r_{2}$, of both the spherical and cubic SPIONs show a trend of increasing values with increasing particles size (Fig. 3a, b).

When compared with spherical SPIONs, the cubic SPIONs show a 1.3- to 2.2-fold increase in longitudinal molar $\left(r_{1}\right)$ relaxivity $\left(r_{1}\right.$ : $0.5 \mathrm{mM}^{-1} \mathrm{~s}^{-1} \quad$ [7-nm sphere]; $1.2 \mathrm{mM}^{-1} \mathrm{~s}^{-1} \quad$ [11-nm sphere]; 
$3.2 \mathrm{mM}^{-1} \mathrm{~s}^{-1} \quad$ [14-nm sphere]; $1.1 \mathrm{mM}^{-1} \mathrm{~s}^{-1} \quad$ [7-nm cubic]; $3.4 \mathrm{mM}^{-1} \mathrm{~s}^{-1}$ [11-nm cubic]; $4.2 \mathrm{mM}^{-1} \mathrm{~s}^{-1}$ [14-nm cubic]; Table 1). Likewise, the cubic SPIONs show 1.7- to 6.3-fold increase in transverse molar $\left(r_{2}\right)$ relaxivity when compared to the spherical SPIONs $\left(r_{2}\right.$ : $0.6 \mathrm{mM}^{-1} \mathrm{~s}^{-1} \quad$ [7-nm sphere]; $9.8 \mathrm{mM}^{-1} \mathrm{~s}^{-1} \quad$ [11-nm sphere]; $40 \mathrm{mM}^{-1} \mathrm{~s}^{-1} \quad$ [14-nm sphere]; $3.8 \mathrm{mM}^{-1} \mathrm{~s}^{-1} \quad$ [7-nm cubic]; $36.8 \mathrm{mM}^{-1} \mathrm{~s}^{-1}$ [11-nm cubic]; $68.8 \mathrm{mM}^{-1} \mathrm{~s}^{-1}$ [14-nm cubic]; Table 1). These differences can be explained by the dominant surfaceto-volume ratio and spin canting effects of the cubic nanoparticles. In short, cubic samples show better $\mathrm{T}_{1^{-}}$and $\mathrm{T}_{2}$-contrast-enhancement compared to their spherical counterparts.

Results show that $\alpha=r_{2} / r_{1}$ ratio increased with increasing nanoparticle size for both spherical and cubic SPIONs ( $\alpha$ : 1.2 [7-nm sphere]; 8.2 [11-nm sphere]; 12.5 [14-nm sphere]; 3.4 [7-nm cubic]; 10.8 [11$\mathrm{nm}$ cubic]; 16.4 [14-nm cubic]; Table 1). Among these six samples, 14$\mathrm{nm}$ cubic SPIONs have higher molar relaxivities, but a high $\alpha$ value of $\alpha=16.4$ at $3 \mathrm{~T}$, which can suffer from $\mathrm{T}_{2}$-related negative signal voids, especially at high iron concentrations. 11-nm cubic and 14-nm sphere samples possess comparable features $\left(\alpha_{11 \mathrm{~nm} \text { (cubic) }}=10.8, \alpha_{14 \mathrm{~nm}}\right.$ (sphere) $=12.5$ at $3 \mathrm{~T}$ ) with higher possibility to show the dual-mode contrast effect. To confirm these claims, we have performed $\mathrm{T}_{1^{-}}$and $\mathrm{T}_{2^{-}}$ weighted contrast enhancement analysis, as shown in Fig. 4. Accordingly, 11-nm cubic and 14-nm sphere SPIONs show the highest SNR and $\mathrm{C}_{\mathrm{Enh}}$ values on $\mathrm{T}_{1}$-weighted images and lowest $\mathrm{SNR}$ and highest $\mathrm{C}_{\mathrm{Enh}}$ values on $\mathrm{T}_{2}$-weighted images, demonstrating their dual-mode contrast effect. It is interesting to note that the 14-nm cubic SPIONs with the highest $r_{1}$ relaxivity did not yield the highest $C_{E n h}$ for $T_{1}$-weighted images. This result was due to the very high $\mathrm{r}_{2}$ relaxivity value of these nanoparticles, which caused a signal reduction even at the relatively short TE of $12 \mathrm{~ms}$ for the $\mathrm{T}_{1}$-weighted images. Hence, 11-nm cubic SPIONs as the smaller particles with higher positive and negative contrast effect are selected as an optimal tracer for synergistic MRI experiment for in-vivo studies performed in this work.

\subsection{Cell survival}

Cellular toxicity of 11-nm silica-coated cubic SPIONs was examined using the L929 mouse cell line with different iron concentrations in $\mathrm{ddH}_{2} \mathrm{O}$. In-vitro visibility of the $\mathrm{L} 929$ cell showed that more than $90 \%$ of the cells survived for $24 \mathrm{~h}$ even at a very high concentration of $100 \mu \mathrm{g} \mathrm{Fe} \mathrm{mL}^{-1}$, indicating negligible toxicity.

\subsection{In-vivo experiments}

In-vivo $\mathrm{T}_{1}$ - and $\mathrm{T}_{2}$-contrast-enhancement analysis performed on the selected ROI at six different time points is summarized in Table 2. The rat was injected with $1 \mathrm{mg} / \mathrm{kg}$ of contrast agent as described in the Methods section. The analysis on $\mathrm{T}_{1}$-weighted images showed that $\mathrm{SNR}$, $\mathrm{CNR}$, and $\mathrm{C}_{\mathrm{Enh}}$ gradually increased as the time progressed, and reached their maximum values at 70-min post-contrast. No significant changes with respect to maximum value (at 70-min post-contrast) were seen at 90-min post-contrast. On the other hand, the analysis on $\mathrm{T}_{2}$-weighted images showed that SNR and CNR decreased as time progressed and reached their lowest values at 70 -min post-contrast. This reduction in SNR and CNR is expected due to the negative contrast effect of SPIONs in $\mathrm{T}_{2}$-weighted images. Importantly, $\mathrm{C}_{\mathrm{Enh}}$ gradually increased to reach a maximum at 70 -min post contrast, indicating the $\mathrm{T}_{2}$-contrast-enhancement capability of the 11-nm silica-coated cubic SPIONs. For both $\mathrm{T}_{1}$-weighted and $\mathrm{T}_{2}$-weighted images, $\mathrm{SNR}, \mathrm{CNR}$, and $\mathrm{C}_{\mathrm{Enh}}$ metrics returned back to values close to their pre-contrast levels at 18-hour postcontrast, which indicates a half-life less than $18 \mathrm{~h}$ for $11-\mathrm{nm}$ cubic SPIONs.

In-vivo $\mathrm{T}_{1}$ - and $\mathrm{T}_{2}$-weighted spin-echo images in rat kidney pre- and 70-min post-injection of the 11-nm silica-coated cubic SPIONs are shown in Figs. 5 and 6, respectively. The first, second, and third rows of the figures show coronal, sagittal, and transversal images from spin- echo acquisitions before and after injection of the contrast agent, respectively. At 70-min post-injection, the kidney region appears brighter on the $\mathrm{T}_{1}$-weighted spin-echo images, demonstrating the positive contrast improvement of the $11-\mathrm{nm}$ cubic SPIONs. The ROI is surrounded by the red dashed circle (Fig. 5). Likewise, abdominal $\mathrm{T}_{2}$-weighted spinecho images taken $70 \mathrm{~min}$ after injection show that kidney turned to darker, demonstrating the negative contrast-enhancement of the 11-nm cubic SPIONs. The negatively-enhanced region that we focused as ROI is marked by the red dashed circle (Fig. 6).

The analysis of the $\mathrm{C}_{\mathrm{Enh}}$ and SNR in the kidney region at 70-min post-injection indicated that contrast is enhanced in the region of interest on both the $\mathrm{T}_{1}$ - and $\mathrm{T}_{2}$-weighted acquisitions. As given in Table 2 , post-injection $\mathrm{C}_{\mathrm{Enh}}$ analysis in the kidney region resulted in $64 \%$ contrast enhancement on $\mathrm{T}_{1}$-weighted transversal images. Also, $\mathrm{C}_{\mathrm{Enh}}$ study reported $48 \%$ contrast enhancement on $\mathrm{T}_{2}$-weighted transversal images. These results demonstrate the feasibility of using the 11-nm cubic SPIONs to synergistically enhance the contrast on both $\mathrm{T}_{1}$ - and $\mathrm{T}_{2}$-weighted in-vivo MR imaging.

\section{Discussion}

The need for a synergistic positive and negative contrast enhancing and safe MRI contrast agent that can be utilized in clinical diagnosis has attracted the interest of many research groups $[18,19]$. The combination of both $\mathrm{T}_{1}$-contrast-enhanced image with high spatial resolution and $\mathrm{T}_{2}$-contrast-enhanced image with increased lesion detection capability allows complementary anatomic and pathologic information [20]. In this study, by controlling the shape and size of the SPIONs, we targeted a new class of synergistic MRI contrast agents. We synthesized two different groups of nanoparticles, in spherical and cubic shapes. Our investigations resulted in two main findings: First, experimental analysis on both the spherical and cubic iron oxides showed an increase of $r_{1}$ and $r_{2}$ as the size increases. More importantly, in comparing the two groups of the synthesized SPIONs, cubic SPIONs showed higher molar relaxivities $\left(r_{1}\right.$ and $\left.r_{2}\right)$ than their spherical counterparts. This observation can be explained by increased surface-to-volume ratio and spin canting effects in the large surfaces and sharp edges of the cubic samples [26,27]. Also, $\alpha=r_{2} / r_{1}$ ratio was larger for the same-sized cubic nanoparticles when compared to spherical nanoparticles, because of the relatively large increases in $r_{2}$. In-vitro contrast enhancement analysis results showed that $11-\mathrm{nm}$ cubic SPIONs can be an optimal sample as a synergistic contrast agent for in-vivo MRI experiments.

Second, as a proof-of-concept feasibility study, we showed that 11$\mathrm{nm}$ cubic SPIONs are promising candidates as a single agent for synergistic contrast in MRI. The results of the in-vivo experiments were in agreement with the in-vitro relaxivity and contrast enhancement analysis of the 11-nm cubic SPIONs. It should be noted that the in-vivo SNR and $\mathrm{C}_{\mathrm{Enh}}$ was lower than the in-vitro case, which could be due to the reduced in-vivo iron concentration in the kidney, as well as environmental differences between the in-vitro and in-vivo cases (such as the $\mathrm{T}_{1}$ and $\mathrm{T}_{2}$ of the in-vitro medium $v$ s. that of the kidney tissue before injection), and differences in pulse sequence parameters.

Gadolinium complexes are the most commonly used $\mathrm{T}_{1}$ contrast agents in MRI. In contrast to SPIONs, however, gadolinium complexes have short blood half-life. As reported in the literature, MR images show high contrast immediately after injection of gadolinium, but this effect vanishes rapidly. The SPIONs are shown to maintain the enhancement of contrast at a high value for more than $1 \mathrm{~h}$, while the gadolinium-enhanced contrast drops within 3 min [15]. This rapid reduction restricts the usage of gadolinium complexes in conjunction with long scan time (e.g., longer than $10 \mathrm{~min}$ ). The contrast enhancement analysis of the proposed cubic SPIONs also showed that the highest contrast level was maintained at a constant level between 70-min and 90-min after injection, providing a wide window for in-vivo imaging. Furthermore, the free gadolinium ions from various labels of gadolinium complexes are known to be very toxic [31,32]. SPIONs, on the 
other hand, are known to have increased biocompatibility and can even be used safely for iron therapy in humans [33].

By taking advantage of both positive and negative contrast effect using a single contrast material, the proposed contrast agent may be used as a promising synergistic contrast tracer. It should be noted that the MR signal intensity and contrast effects are dependent on image acquisition parameters, particularly TR, TE and flip angle [22], as well as the magnetic property of contrast materials. This work did not aim to optimize the imaging parameters, but rather focused on optimizing the nanoparticles based on their size and shape characteristics. Accordingly, the in-vivo experiments were geared towards showing the feasibility of the 11-nm cubic SPIONs as a dual-mode contrast agent. Investigating the pharmacodynamics and pharmacokinetic effects of these nanoparticles on different organs using complementary in-vivo MRI experiments remains as important future work.

\section{Conclusion}

In this study, by controlling the shape and size of SPIONs, we have synthesized a new class of synergistic (dual-mode) MRI contrast agent. The in-vitro contrast enhancement analysis showed that the synthesized 11-nm cubic SPIONs with small size have high dual-contrast effect, suitable for use during in-vivo imaging. Results of the toxicity analysis showed that the antagonistic effects of the 11-nm cubic SPIONs on the L929 line cell were very low even at high concentrations of the contrast agent. The feasibility of the synergistic contrast effect was investigated in-vivo on a 3T MRI scanner, demonstrating high levels of contrast enhancement 70-min post-injection. These results demonstrate the promising potential of the synthesized 11-nm silica-coated cubic SPIONs as a synergistic MRI contrast agent.

\section{Acknowledgment}

We gratefully acknowledge Dr. Gamze Aykut, Department of Molecular Biology and Genetics, Bilkent University, Ankara, Taner Demir and Dr. Volkan Acikel, Bilkent University, Ankara, for their assistance in in-vivo experiments, and all UMRAM staff for providing technical support. The authors also gratefully thank scientific discussion of Dr. Tolga Cukur. We would like to acknowledge Biomaten (METU) for the cytotoxicity experiments.

\section{References}

[1] Hoult DI, Lauterbur PC. The sensitivity of the zeugmatographic experiment involving human samples. J Magn Reson 1979;34:425-33.

[2] Wright GA. Magnetic resonance imaging. IEEE Signal Process Mag 1997;14:56-66.

[3] Clarke SE, Weinmann HJ, Dai E, Lucas AR, Rutt BK. Comparison of two blood pool contrast agents for 0.5-T MR angiography: experimental study in rabbits. Radiology 2000;214:787-94.

[4] Thorek DL, Ulmert D, Diop NF, Lupu ME, Doran MG, Huang R, et al. Non-invasive mapping of deep-tissue lymph nodes in live animals using a multimodal PET/MRI nanoparticle. Nat Commun 2014;5:3097. http://dx.doi.org/10.1038/ncomms4097.

[5] Klug G, Kampf T, Bloemer S, Bremicker J, Ziener CH, Heymer A, et al. Intracellular and extracellular $\mathrm{T}_{1}$ and $\mathrm{T}_{2}$ relaxivities of magneto-optical nanoparticles at experimental high fields. Magn Reson Med 2010;64(6):1607-15. http://dx.doi.org/ $10.1002 / \mathrm{mrm} .22557$.

[6] Lee S, Kim B, Na B, Hyeon T. Paramagnetic inorganic nanoparticles as $\mathrm{T}_{1}$ MRI contrast agents. WIREs Nanomed Nanobiotechnol 2014;6:196-209. http://dx.doi. org/10.1002/wnan.1243.

[7] Zachary RS, Forrest M, Kievit A, Zhang M. Magnetite nanoparticles for medical MR imaging. Mater Today 2011;14:330-8. http://dx.doi.org/10.1016/S1369-7021(11) 70163-8.

[8] Au-Hui LU, Salabas EL, Schuth F. Magnetic nanoparticles: synthesis, protection, functionalization, and application. Angew Chem 2007;46:1222-44. http://dx.doi. org/10.1002/anie.200602866.

[9] Huber ME, Paetsch I, Schnackenburg B, Bornstedt A, Nagel E, Fleck E, et al.
Performance of a new gadolinium-based intravascular contrast agent in freebreathing inversion-recovery 3D coronary MRA. Magn Reson Med 2003;49:115-21. http://dx.doi.org/10.1002/mrm.10350.

[10] Kuo PH, Kanal E, Abu-Alfa AK, Cowper SE. Gadolinium-based MR contrast agents and nephrogenic systemic fibrosis. Radiology 2007;242:647-9. http://dx.doi.org/ 10.1148/radiol.2423061640.

[11] Ziener CH, Bauer WR, Jakob PM. Transverse relaxation of cells labeled with magnetic nanoparticles. Magn Reson Med 2005;54(3):702-6. http://dx.doi.org/10. 1002/mrm.20634.

[12] Yi-Xiang JW, Shouhu X, Marc P, Jean-Marc I. Recent advances in superparamagnetic iron oxide nanoparticles for cellular imaging and targeted therapy research. Curr Pharm Des 2013;19(37):6575-93.

[13] Sharifi S, Seyednejad H, Laurent S, Atyabi F, Saei A, Mahmoudi M. Superparamagnetic iron oxide nanoparticles for in vivo molecular and cellular imaging. Contrast Media Mol Imaging 2015;10:329-55. http://dx.doi.org/10. 1002/cmmi.1638.

[14] Chris VB, Xiaowei Z, George S, Paula JG, Brian KR. Application of the static dephasing regime theory to superparamagnetic iron-oxide loaded cells. Magn Reson Med 2002;48:52-61. http://dx.doi.org/10.1002/mrm.10192.

[15] Byung HK, Nohyun L, Hyoungsu K, Hyoungsu K, Kwangjin A, Yong P. Large-scale synthesis of uniform and extremely small-sized iron oxide nanoparticles for highresolution T1 magnetic resonance imaging contrast agents. J Am Chem Soc 2011;133:12624-31. http://dx.doi.org/10.1021/ja203340u.

[16] Lee N, Choi Y, Lee Y, Park M, Moon WK, Choi SH, et al. Water-dispersible ferrimagnetic iron oxide nanocubes with extremely high $\mathrm{r} 2$ relaxivity for highly sensitive in vivo MRI of tumors. Nano Lett 2012;12(6):3127-31. http://dx.doi.org/10. $1021 / \mathrm{nl} 3010308$.

[17] Lee CM, Jeong HJ, Kim EM, Kim DW, Lim ST, Kim HT, et al. Superparamagnetic iron oxide nanoparticles as a dual imaging probe for targeting hepatocytes in vivo. Magn Reson Med 2009;62(6):1440-6. http://dx.doi.org/10.1002/mrm.22123.

[18] Girard OM, Du J, Agemy L, Sugahara KN, Kotamraju VR, Ruoslahti E, et al. Optimization of iron oxide nanoparticle detection using ultrashort echo time pulse sequences: comparison of $\mathrm{T}_{1}, \mathrm{~T}_{2}{ }^{*}$, and synergistic $\mathrm{T}_{1}-\mathrm{T}_{2}{ }^{*}$ contrast mechanisms. Magn Reson Med 2011;65:1649-60. http://dx.doi.org/10.1002/mrm.22755.

[19] Bae KH, Kim YB, Lee Y, Hwang JY, Park HW, Park TG. Bioinspired synthesis and characterization of gadolinium-labeled magnetite nanoparticles for dual contrast T1-and T2-weighted magnetic resonance imaging. Bioconjug Chem 2010;21(3):505-12. http://dx.doi.org/10.1021/bc900424u.

[20] Sharma VK, Alipour A, Soran-Erdem Z, Kelestemur Y, Aykut ZG, Demir HV. Fluorescent heterodoped nanotetrapods as synergistically enhancing positive and negative MRI contrast agents. ACS Appl Mater Interfaces 2016;8(19):12352-9. http://dx.doi.org/10.1021/acsami.6b02407.

[21] Zijian Z, Dengtong H, Jianfeng B, Qiaoli C, Gang L, Zhong C, et al. A synergistically enhanced $\mathrm{T}_{1}-\mathrm{T}_{2}$ dual-modal contrast agent. Adv Mater 2012;24:6223-8. http://dx. doi.org/10.1002/adma.201203169.

[22] Jung H, Park B, Lee C, Cho J, Suh J, Park J, et al. Dual MRI $T_{1}$ and $\mathrm{T}_{2}$ * contrast with size-controlled iron oxide nanoparticles. Nanomedicine 2014;10:1679-89. http:// dx.doi.org/10.1016/j.nano.2014.05.003.

[23] Kwon H, Shim W, Cho G, Cho HJ, Jung HS, Lee CK, et al. Simultaneous evaluation of vascular morphology, blood volume and transvascular permeability using SPIONbased, dual-contrast MRI: imaging optimization and feasibility test. NMR Biomed 2015;28(6):624-32. http://dx.doi.org/10.1002/nbm.3293.

[24] Bhatti ML, Marin D, Nelson R. Emerging applications for ferumoxytol as a contrast agent in MRI. J Magn Reson Imaging 2015;41(4):884-98. http://dx.doi.org/10. 1002/jmri.24691.

[25] Berry CC, Curtis AG. Functionalisation of magnetic nanoparticles for applications in biomedicine. J Phys D Appl Phys 2003;36:198-206.

[26] Batlle X, Labarta A. Finite-size effects in fine particles: magnetic and transport properties. J Phys D Appl Phys 2002;35:15-42.

[27] Zhenghuan Z, Zijian Z, Jianfeng B, Wang Z, Hu J, Chi X, et al. Octapod iron oxide nanoparticles as high performance T2 contrast agents for magnetic resonance imaging. Nat Commun 2013;3266. http://dx.doi.org/10.1038/ncomms3266.

[28] Koenig SH, Kellar KE. Theory of 1/T1 and 1/T2 NMRD profiles of solutions of magnetic nanoparticles. Magn Reson Med 1995;34(2):227-33.

[29] Buxton RB, Edelman RR, Rosen BR, Wismer GL, Brady TJ. Contrast in rapid MR imaging- $\mathrm{T}_{1}$-weighted and $\mathrm{T}_{2}$-weighted imaging. J Comput Assist Tomogr 1987;11:7-16.

[30] Rock A, Muller RN, Gillis P. Thery of proton relaxation induced by super paramagnetic particles. J Chem Phys 1999;110:5403-11.

[31] Penfield JG, Reilly RF. What nephrologists need to know about gadolinium. Nat Clin Pract Nephrol 2007;3(12):654-68. http://dx.doi.org/10.1038/ncpneph0660.

[32] Warsi MF, Adams RW, Duckett SB, Chechik V. Gd-functionalised Au nanoparticles as targeted contrast agents in MRI: relaxivity enhancement by polyelectrolyte coating. Chem Commun 2010;21;46(3):451-3. http://dx.doi.org/10.1039/ b915223g.

[33] Lu M, Cohen MH, Rieves D, Pazdur R. FDA report: ferumoxytol for intravenous iron therapy in adult patients with chronic kidney disease. Am J Hematol 2010;85:315-9. http://dx.doi.org/10.1002/ajh.21656. 